\title{
Right atrial and ventricular strain detects subclinical changes in right ventricular function in precapillary pulmonary hypertension
}

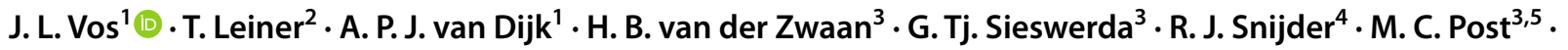

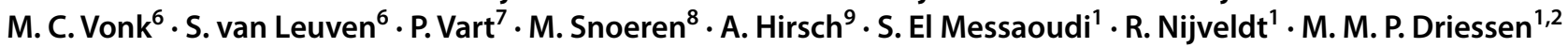

Received: 23 November 2021 / Accepted: 4 February 2022 / Published online: 21 February 2022

(c) The Author(s) 2022

\begin{abstract}
Right ventricular (RV) ejection fraction (EF) by cardiac magnetic resonance (CMR) correlates to outcome in precapillary pulmonary hypertension (pPH) patients, but is insensitive to early changes. Strain might provide incremental information. In this study, we compare right atrial (RA) and RV strain in $\mathrm{pPH}$ patients to healthy controls, and evaluate the prognostic value of strain in pPH. In this cross-sectional study, 45 pPH patients and 20 healthy controls underwent CMR, and feature-tracking derived RA and RV strain were evaluated. $\mathrm{pPH}$ patients had impaired RA reservoir and conduit strain, and RV longitudinal strain (LS), compared to healthy controls. In pPH patients with preserved RVEF ( $\geq 50 \%, \mathrm{n}=18)$, RA reservoir $(35 \% \pm 9$ vs. $41 \% \pm 6, \mathrm{p}=0.02)$ and conduit strain $(16 \% \pm 8$ vs. $23 \% \pm 5, \mathrm{p}=0.004)$, and RV-LS $(-25 \% \pm 4$ vs. $-31 \% \pm 4, \mathrm{p}<$ 0.001 ) remained impaired, compared to healthy controls. The association of strain with the primary endpoint (combination of all-cause death, lung transplantation, and heart failure hospitalization) was evaluated using a multivariable Cox regression model. RV-LS (HR 1.18, 95\%-CI 1.04-1.34, p = 0.01) and RA strain (reservoir: HR 0.87, 95\%-CI 0.80-0.94, p = 0.001; conduit: HR 0.85, 95\%-CI 0.75-0.97, $\mathrm{p}=0.02$, booster: HR 0.81, 95\%-CI 0.71-0.92, $\mathrm{p}=0.001$ ) were independent predictors of outcome, beyond clinical and imaging features. In conclusion, pPH patients have impaired RA strain and RV-LS, even when RVEF is preserved. In addition, RA strain and RV-LS were independent predictors of adverse prognosis. These results emphasize the incremental value of RA and RV strain analyses, to detect alterations in RV function, even before RVEF declines.
\end{abstract}

Keywords Pulmonary hypertension $\cdot$ Cardiovascular magnetic resonance imaging $\cdot$ Feature tracking $\cdot$ Right ventricular dysfunction

\section{Introduction}

Precapillary pulmonary hypertension $(\mathrm{pPH})$ causes increased right ventricular (RV) afterload, inducing RV remodeling. The prognosis of patients with $\mathrm{PH}$ is not

M. M. P. Driessen

Mieke.Driessen@radboudumc.nl

1 Department of Cardiology, Radboud University Medical Center, Geert Grooteplein 10, 6525 GA Nijmegen, The Netherlands

2 Department of Radiology, University Medical Center Utrecht, Utrecht, The Netherlands

3 Department of Cardiology, University Medical Center Utrecht, Utrecht, The Netherlands

4 Department of Pulmonology, St. Antonius Hospital, Nieuwegein, The Netherlands solely determined by pulmonary arterial pressure or pulmonary vascular resistance. Various studies have shown that the ability of the RV to adequately adapt to increased pressure loading is essential for a patient's prognosis [1, 2]. If $\mathrm{RV}$ adaptation is not adequate, $\mathrm{pPH}$ will often result

5 Department of Cardiology, St. Antonius Hospital, Nieuwegein, The Netherlands

6 Department of Rheumatology, Radboud University Medical Center, Nijmegen, The Netherlands

7 Department of Health Evidence, Radboud University Medical Center, Nijmegen, The Netherlands

8 Department of Radiology, Radboud University Medical Center, Nijmegen, The Netherlands

9 Department of Cardiology and Radiology and Nuclear Medicine, Erasmus Medical Center, University Medical Center Rotterdam, Rotterdam, The Netherlands 
in RV dysfunction and dilatation, heart failure, and ultimately death. Right atrial (RA) dilatation and functional decline, caused by increased RV end-diastolic pressure and tricuspid regurgitation, is also associated with prognosis [1]. Cardiac magnetic resonance (CMR) imaging is an accurate and reproducible tool to assess RV morphology, volumes and ejection fraction (EF) and is considered the gold standard for noninvasive assessment of RV function $[1,3,4]$. However, RVEF is a global measure of RV function, and a decline in RVEF occurs at a late stage of pPH, when RV remodeling (hypertrophy and increasing contractility) falls short. Therefore, it is important to understand and recognize changes in RV function that occur before RVEF starts to decline [1]. CMR feature tracking (FT) is a promising post-processing technique that allows the assessment of changes in myocardial deformation on standard cine images [5], potentially useful to detect both global and segmental alterations in RA and RV function.

The aim of this study, therefore, is to evaluate RA and RV strain in relation to volume and function in $\mathrm{pPH}$ patients, in order to investigate whether alterations in myocardial deformation precede a decrease in RVEF, and to evaluate their prognostic importance.

\section{Methods}

In this cross-sectional study, we included prevalent patients with $\mathrm{pPH}$, consecutively enrolled in two different studies. The first study included pPH patients $(n=33)$ enrolled between August 2012 and November 2013 [6, 7]. The second study included patients with pulmonary arterial hypertension associated with systemic sclerosis enrolled between August 2019 and January $2020(\mathrm{n}=12)$. In- and exclusion criteria were similar in both studies. The diagnosis of $\mathrm{pPH}$ was previously established on a cardiac catheterization, in accordance with the ESC/ESR guidelines [3]. Patients were recruited from three tertiary hospitals in the Netherlands. A group of healthy subjects of similar age and gender served as a control group $(n=20)$. The Ethical Review boards of all participating centers approved the study. Written informed consent was obtained from all study participants prior to inclusion. Demographic data and CMR were obtained in all subjects. Transthoracic echocardiography (TTE) and CMR were performed on the same day. TTE was only performed in the $\mathrm{pPH}$ patients. In addition, follow-up data was collected of $\mathrm{pPH}$ patients included in the first study $(\mathrm{n}=33)$, making use of medical records. The primary endpoint was a composite endpoint of any major adverse cardiovascular event (MACE), defined as the combination of all-cause mortality, lung transplantation, and heart failure hospitalization. In case more than one endpoint in the same patient occurred, the most severe endpoint was used (death > lung transplantation $>$ heart failure hospitalization).

\section{Echocardiography acquisition}

The TTE was performed with a Toshiba Artida system (Toshiba) with a 5-MHz transducer, or a GE medical systems VIVID E9 (GE Healthcare) with a $1.5-4 \mathrm{MHz}$ phased array transducer. Offline analysis was performed on commercially available software. RV systolic pressure (RVSP) was calculated by adding the Bernoulli equation derived pressure gradient from the maximum tricuspid regurgitation velocity to the estimated RA pressure [8]. RV fractional area change (FAC), defined as ([end-diastolic area - end-systolic area]/end-diastolic) $\times 100 \%$, and tricuspid annular plane systolic excursion (TAPSE) by M-mode echocardiography, were measured on the apical 4-chamber view.

\section{CMR imaging acquisition}

All study participants were scanned on commercially available 1.5 Tesla MR-scanners (Ingenia R4.1.2, Philips Healthcare; Siemens Avanto, Siemens Healthcare, GmbH; Signa or Discovery, GE medical systems). Standard cine images were acquired during repeated end-expiratory breath holds, using a balanced steady-state free precession sequence. Cine images were acquired with similar spatial resolution (typical voxel size $1.5 \times 1.5 \times 5-8 \mathrm{~mm}^{3}$, repetition time/echo time $=3.2 / 1.6$, flip angle $>60^{\circ}$ ), and temporal resolution (30 phases per cardiac cycle, except for the long axis cine images in 3 study participants; in whom 25 phases per cardiac cycle, typically $25-30 \mathrm{~ms}$ ). Consecutive short-axis cine images were acquired every $10 \mathrm{~mm}$ from base to apex up to measure RV (including the trabeculae) [6] and LV volumes and calculate EF.

\section{CMR feature tracking analysis}

RV strain was measured using Medis Qstrain software (Medis Medical Imaging Systems, version 2.0.48.8.) on standard cine images. Endocardial contours were manually drawn in end-systolic and end-diastolic frame (defined as the smallest and largest RV volume, respectively), and automatically tracked in all other consecutive frames. Strain is computed by measuring the change in endocardial length between end-systolic and end-diastolic phase (as a percentage, with the end-diastolic length as reference) in a certain direction: Longitudinal strain (LS) in the longitudinal axis, circumferential strain (CS) when it is measured along the circumference. Global LS (GLS) was measured on the 4-chamber cines. CS was measured on the basal-, mid-, and apical 
ventricular short-axis cines, the average (global CS; GCS) is automatically calculated by Qstrain. RV contraction time was defined as time to peak strain (TTP). GCS was divided by GLS to assess GCS/GLS ratio. To detect mechanical discoordination, segmental LS (free wall-LS and septal-LS), and intraventricular delay (free wall TTP minus septal TTP) was analyzed. RA strain was measured on 4-chamber cines, and the reservoir (collecting the central venous return), conduit (passive filling of blood to the RV during early and mid-diastole) and booster strain (atrial contraction; active, late diastolic phase of the RV) was analyzed. RA volumes and EF, using the biplane Simpson's area-length method, are automatically generated by Qstrain software [9]. RV LS and RA strain parameters are illustrated in Fig. 1. Strain analyses were performed by one single investigator (JV), supervised by a level III CMR-physician with $>15$ years of experience (RN). To evaluate intraobserver (performed by JV, 2 weeks after the first analysis) and interobserver (performed by a second investigator, MD) variability, strain analyses were repeated in 30 CMR scans (10 scans of healthy controls, 20 scans of $\mathrm{pPH}$ patients).

\section{Statistical analyses}

Continuous variables are presented as mean \pm standard deviation (if normally distributed) or median (interquartile range) if not normally distributed. Categorical variables are presented as total number (percentage). Normality was assessed visually using Q-Q-plots and histograms. pPH patients and control group are compared using the independent sample t-test for normally distributed continuous data, or the Mann-Whitney U for not normally distributed continuous data. The Chi-Square test was used to compare categorical variables between groups. The intraclass correlation coefficient was used to measure inter- and intra-observer variability.

A sub analysis was performed to analyze whether FT can detect differences between healthy controls and $\mathrm{pPH}$ patients with a still preserved RVEF. A preserved RVEF is defined as RVEF $\geq 50 \%$ [10]. To analyze changes in RA and $\mathrm{RV}$ contraction in different stages of RV function, $\mathrm{pPH}$ patients were further divided into groups according to the RVEF (RVEF $\geq 50 \%$, RVEF 40-50\%, and RVEF $<40 \%$ ) to get equally sized groups. A one-way analysis of variance (ANOVA) test or Kruskal Wallis test was used for comparison between healthy controls and the $\mathrm{pPH}$ groups stratified by RVEF. When ANOVA was significant, a post-hoc analysis was performed for pairwise comparison. Type I error rate was controlled using Dunnett's test for multiple testing. Unadjusted and adjusted cox proportional hazards regression analyses were performed to evaluate the hazard ratio (HR, with 95\% confidence interval; CI) of strain parameters. Strain parameters are included as continuous parameters.
A selection of covariates that are previously suggested to be of clinical relevance (sex, age, WHO functional class, estimated RVSP, RVEF, and indexed RV end-diastolic volume $[2,11,12])$ were first univariably tested, and when significant added to the adjusted model. All statistical analyses were performed using SPSS (version 25). A P value less than 0.05 was considered statistically significant.

\section{Results}

\section{Study population}

In total, 20 healthy controls [aged 56 years (52-59), 20\% male] and 45 patients with pPH [aged 58 years (46-72), $24 \%$ male] were included, characteristics shown in Table 1. In one $\mathrm{pPH}$ patient the CMR was not performed on the same day as TTE (15 days difference).

\section{Echocardiographic and CMR parameters}

The mean estimated RVSP was $56 \pm 19 \mathrm{mmHg}$ in $\mathrm{pPH}$ patients (Table 2). To be noted, RVSP could not be estimated due to the lack of tricuspid regurgitation in $2 \mathrm{pPH}$ patients. Due to poor image quality, FAC could not be measured in 11 patients $(24 \%)$.

CMR derived LV volumes and EF were similar in $\mathrm{pPH}$ patients and healthy controls, whereas RVEF was impaired compared to healthy controls (Table 2). In addition, $\mathrm{pPH}$ patients had larger end-diastolic and end-systolic volumes, and higher RV mass. RA maximum and minimum volumes were higher in $\mathrm{pPH}$ patients (see Table 2 for values).

\section{RA and RV strain analysis}

\section{Reproducibility of RV strain parameters}

The inter- and intra-oberserver variability of strain parameters (GLS and CS) were excellent, with intraclass correlation coefficients ranging from 0.84 to 0.94 (Table S1).

\section{pPH patients versus healthy controls}

Compared to healthy controls, pPH patients had lower RA reservoir and conduit strain, whereas RA booster strain was similar (see Table 3 for values). In one patient CS could not be measured (poor image quality). GLS and regional LS were significantly impaired in $\mathrm{pPH}$ patients compared to healthy controls. GCS, mid-CS and apical-CS were impaired in $\mathrm{pPH}$ patients compared to healthy controls, whereas 


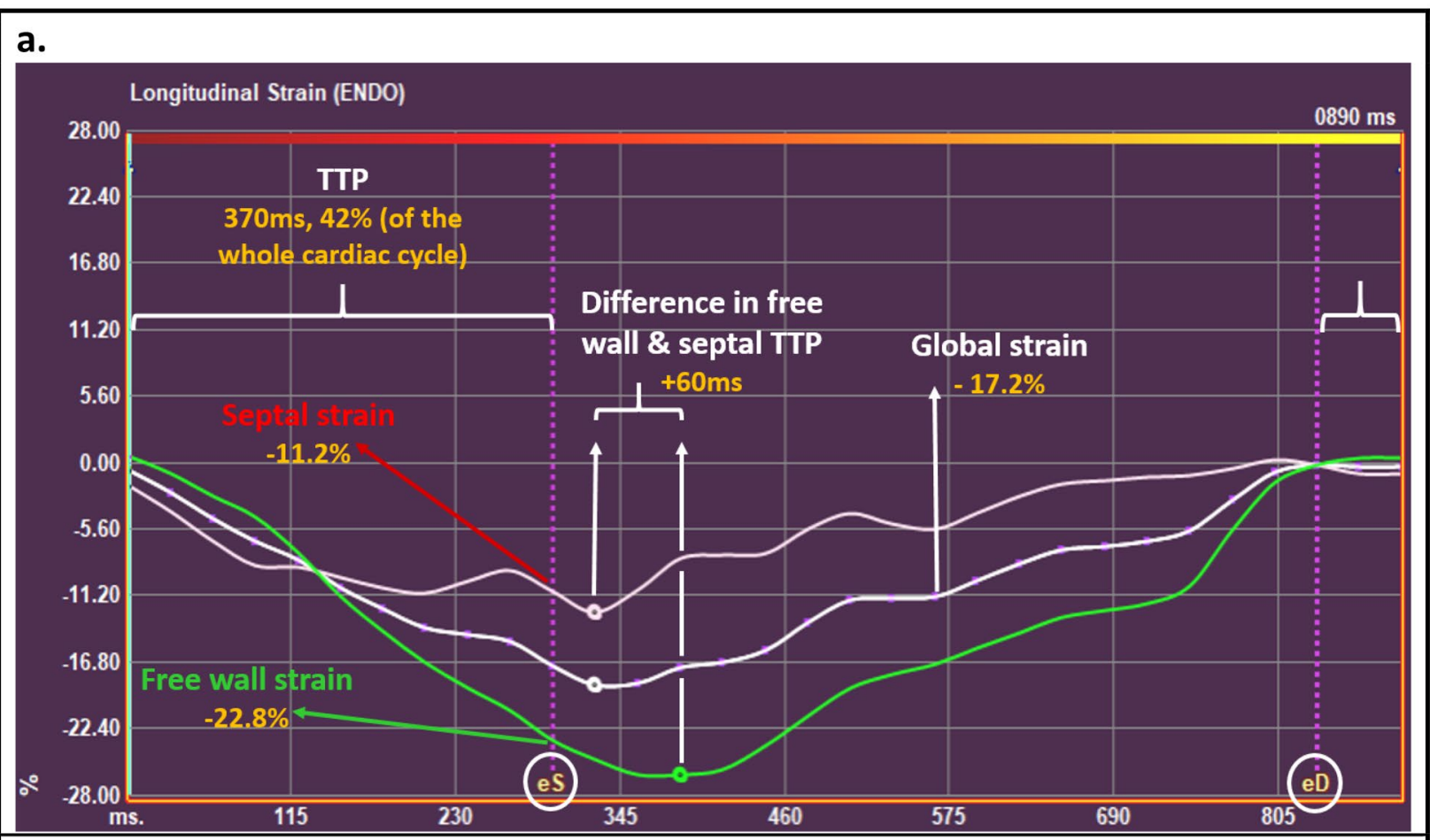

b.

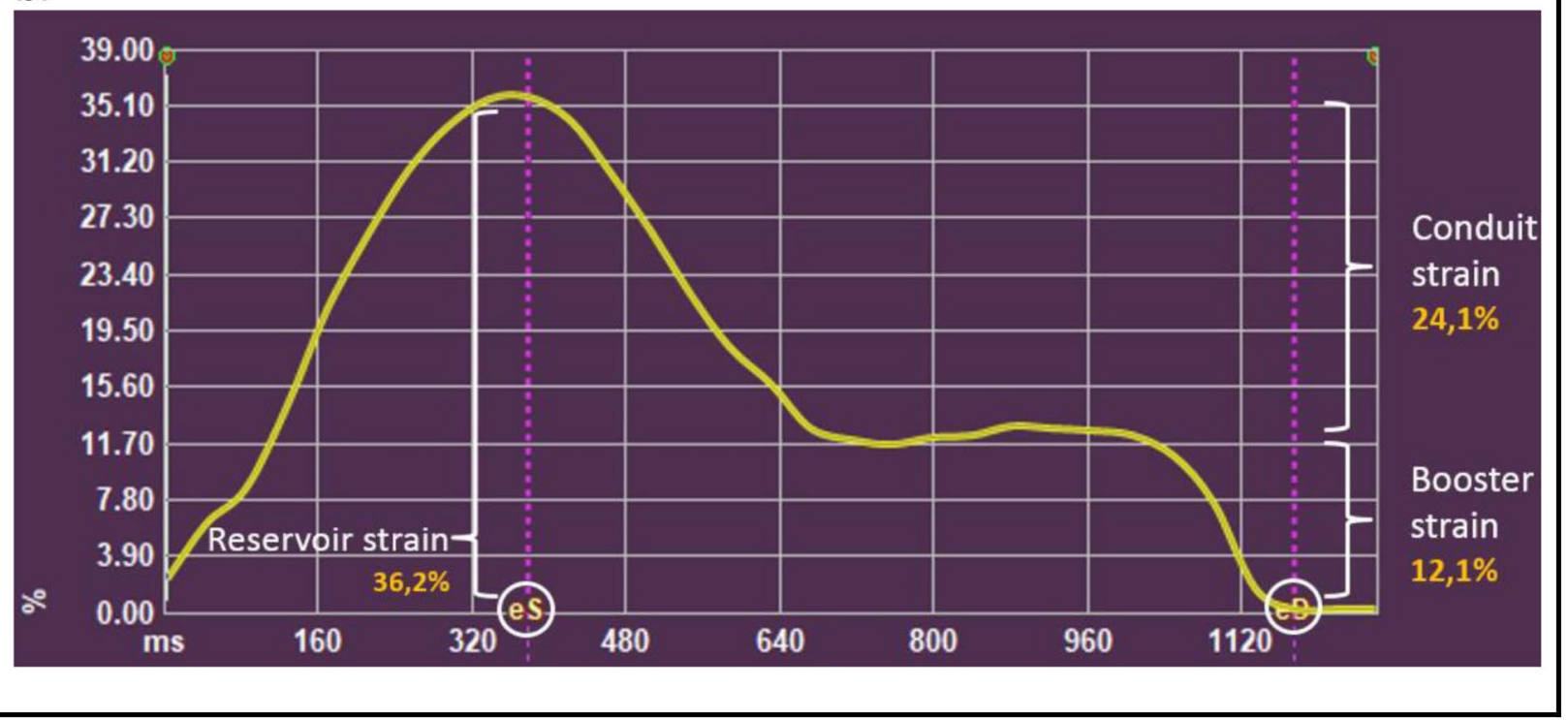

Fig. 1 Depiction of the different strain parameters using Medis Qstrain software. a Right ventricular longitudinal strain parameters. Depiction of right ventricular (RV) longitudinal strain (\%, y-axis) in time (ms, $\mathrm{x}$-axis) in a precapillary pulmonary hypertension patient. Global, free wall and septal strain is measured at the time of eS ('systolic strain'). eD is used as a reference to measure TTP. b Right atrial strain parameters. Depiction of right atrial (RA) strain (\%,y-axis) in

basal-CS was similar in pPH patients and healthy controls. Compared to healthy controls, pPH patients had longer TTP contraction ( $\%$ of the whole cardiac cycle). Figure 2 shows time (ms, $\mathrm{x}$-axis). Reservoir strain measures the expansion of the RA (during RV systole) from the central venous return. Conduit strain is the passive filling of blood from the RA to the RV during early and mid-diastole. Booster strain measures the atrial contraction in the late-diastolic, active, RV filling phase. (eD) end-diastole; (eS) endsystole; TTP time to peak strain

representative images of the RV and RA strain analysis in a healthy control and a pPH patient. 
Table 1 Baseline characteristics of healthy controls and precapillary pulmonary hypertension patients

\begin{tabular}{|c|c|c|c|}
\hline & $\begin{array}{l}\text { Healthy controls } \\
(\mathrm{n}=20)\end{array}$ & $\mathrm{pPH}$ patients $(\mathrm{n}=45)$ & $P$ value \\
\hline \multicolumn{4}{|l|}{ Demographics } \\
\hline Age (years) & $56[52-59]$ & $58[46-72]$ & 0.34 \\
\hline Male, $n(\%)$ & $4(20 \%)$ & $11(24 \%)$ & 0.76 \\
\hline Body surface area $\left(\mathrm{m}^{2}\right)$ & $1.8 \pm 0.2$ & $1.9 \pm 0.3$ & 0.09 \\
\hline \multicolumn{4}{|l|}{ Clinical classification of $\mathrm{PH}, n(\%)$} \\
\hline Idiopathic PAH & & $15(33 \%)$ & \\
\hline PAH associated with systemic sclerosis & & $12(27 \%)$ & \\
\hline СТЕPH & & $18(40 \%)$ & \\
\hline Duration of disease (years) & & $5.3 \pm 5.4$ & \\
\hline \multicolumn{4}{|l|}{ Treatment of PH, $n(\%)$} \\
\hline None & & $2(4 \%)$ & \\
\hline Monotherapy & & $15(33 \%)$ & \\
\hline Dual therapy & & $21(47 \%)$ & \\
\hline Triple therapy & & $7(16 \%)$ & \\
\hline Endotheline receptor antagonists & & $38(64 \%)$ & \\
\hline Prostanoids & & $9(20 \%)$ & \\
\hline Phosphodiesterase inhibitors & & $29(64 \%)$ & \\
\hline Riociguat & & $2(4 \%)$ & \\
\hline Oxygen therapy & & $9(20 \%)$ & \\
\hline \multicolumn{4}{|l|}{ Functional status assessment } \\
\hline \multicolumn{4}{|l|}{ WHO Functional Class, $n(\%)$} \\
\hline I & & $3(7 \%)$ & \\
\hline II & & $29(64 \%)$ & \\
\hline III & & $12(27 \%)$ & \\
\hline IV & & $1(1 \%)$ & \\
\hline 6 min walk distance $(\mathrm{m})^{\mathrm{a}}$ & & $451[391-528]$ & \\
\hline NT-proBNP (pg/mL) & & 240 [111-1539] & \\
\hline
\end{tabular}

Values are in means $\pm \mathrm{SD}$, medians [interquartile range], or $\mathrm{n}(\%)$

$A C E$ angiotensin-converting enzyme, ARBs angiotensin II receptor blockers, $C T E P H$ chronic thromboembolic pulmonary hypertension, ERAs endothelin receptor antagonists, PDIs phosphodiesterase inhibitors, $P A H$ pulmonary arterial hypertension, $\mathrm{pPH}$ precapillary pulmonary hypertension

${ }^{\mathrm{a}} \mathrm{n}=43$ (not available in 2 patients)

\section{pPH patients with a preserved RVEF versus healthy controls}

In total, $18 \mathrm{pPH}$ patients had preserved RVEF ( $\mathrm{RVEF} \geq 50 \%)$. RA reservoir and RA conduit strain were lower in $\mathrm{pPH}$ patients, whereas booster strain was preserved (Table 3). GLS $(-25 \% \pm 4$ vs. $-31 \% \pm 4$, p < 0.001$)$ was decreased, and TTP contraction longer in $\mathrm{pPH}$ patients with preserved RVEF compared to healthy controls. Compared to healthy controls, pPH patients still had a preserved GCS, consequently leading to a higher CS/LS ratio. Interestingly, $\mathrm{pPH}$ patients had significantly better basal-CS compared to healthy controls $(-15 \% \pm 3$ vs. $-12 \% \pm 3, p=0.001)$, and there was a trend toward worse apical-CS $(-17 \% \pm 7$ vs. $-21 \% \pm 7, p=0.09$ ). In a multivariate analysis (including indexed-RV end-diastolic and end-systolic volumes, and RVEF), strain parameters were still independently associated with pPH (Table S2). The individual strain values of healthy controls and pPH patients are shown in Table S3. Figure 3 shows main RA and RV strain parameters in $\mathrm{pPH}$ patients stratified in groups by RVEF, compared to healthy controls.

\section{The prognostic value of RA en RV strain to predict MACE in $\mathrm{pPH}$ patients}

During a median follow-up of 8 [7-9] years, 12 (36\%) out of the $33 \mathrm{pPH}$ patients included in the first study reached the primary endpoint [all-cause death $(n=9)$, lung transplantation $(n=2)$, and heart failure hospitalization $(n=1)]$. Only one patient, after $>3$ years of follow up, was lost-tofollow up. All RV and RA strain parameters were associated with MACE, except for the RV CS parameters (for values see Table 4). After adjustment for the univariably significant covariates (WHO functional class $\geq 3$ and indexed RV 
Table 2 Echocardiographic \& CMR parameters of healthy controls \& precapillary pulmonary hypertension patients

Healthy controls $(n=20) \quad$ pPH patients $(n=45) \quad$ P value* $\quad$ pPH patients with pre- P value* served RVEF $(n=18)$

Echocardiography

TAPSE (mm)

$\operatorname{RVSP}(\mathrm{mmHg})^{\mathrm{a}}$

Fractional area change $(\%)^{\mathrm{b}}$

Cardiac magnetic resonance imaging

LVEDV (mL)

LVEDV-indexed $\left(\mathrm{mL} / \mathrm{m}^{2}\right)$

LVESV (mL)

LVESV-indexed $\left(\mathrm{mL} / \mathrm{m}^{2}\right)$

LVEF (\%)

RVEDV (mL)

RVEDV-indexed $\left(\mathrm{mL} / \mathrm{m}^{2}\right)$

RVESV (mL)

RVESV-indexed $\left(\mathrm{mL} / \mathrm{m}^{2}\right)$

RVEF (\%)

$\mathrm{RV}$ mass (g)

$\mathrm{RV}$ mass-indexed $\left(\mathrm{g} / \mathrm{m}^{2}\right)$

RV fractional area change (\%)

RA maximum volume $(\mathrm{mL})$

RA minimum volume $(\mathrm{mL})$

RA volume prior to atrial contraction $(\mathrm{mL})$

RA EF (\%)

$$
\begin{aligned}
& 20 \pm 4 \\
& 56 \pm 19 \\
& 31 \pm 12
\end{aligned}
$$

$136 \pm 25$
$77 \pm 11$
$55 \pm 14$
$31 \pm 6$
$60 \pm 4$
$137[117-163]$
$81[67-87]$
$60 \pm 20$
$33 \pm 10$
$58[54-62]$
$17 \pm 4$
$10 \pm 2$
$50 \pm 5$
$85[73-113]$
$47[32-59]$
$71[55-84]$
$49 \pm 5$

$146 \pm 35$

$146 \pm 35$
$78 \pm 16$
$62 \pm 23$
$33 \pm 11$
$58 \pm 9$
$193[172-273]$
$101[86-138]$
$140 \pm 80$
$76 \pm 50$
$46[38-53]$
$36 \pm 10$
$19 \pm 9$
$36 \pm 10$
$127[99-164]$
$71[54-112]$
$105[81-137]$
$39 \pm 12$

0.28

0.79

0.25

$0.41 \quad 30 \pm 11$

$21 \pm 3$

$44 \pm 11$

$38 \pm 11$

$0.40 \quad 63 \pm 6$

$<\mathbf{0 . 0 0 1} 174$ [133-203]

$<\mathbf{0 . 0 0 1} 94$ [76-101]

$<0.001 \quad 85 \pm 33$

$<0.00144 \pm 13$

$<\mathbf{0 . 0 0 1} 53$ [50-57]

$<0.00127 \pm 10$

$<0.00114 \pm 4$

$<0.00144 \pm 7$

$<\mathbf{0 . 0 0 1} 99$ [84-131]

$<\mathbf{0 . 0 0 1} 54$ [45-85]

$<\mathbf{0 . 0 0 1} 82$ [71-114]

$<0.001 \quad 44 \pm 10$
0.15

0.46

0.80

0.74

0.05

$\mathbf{0 . 0 3}$

0.02

0.006

0.006

$<0.001$

$<0.001$

$<0.001$

$<0.001$

0.08

0.04

0.04

0.07

P-values in bold are statistically significant $(\mathrm{p}<0.05)$

Values are in means $\pm \mathrm{SD}$, medians [interquartile range], or $\mathrm{n}(\%)$

$E D V$ end-diastolic volume, $E F$ ejection fraction, $E S V$ end-systolic volume, $L V$ left ventricular, $p P H$ precapillary pulmonary hypertension, $R A$ right atrium, $R V$ right ventricular, RVSP right ventricular systolic pressure, TAPSE tricuspid annular plane systolic excursion

*Compared to healthy controls

${ }^{\mathrm{a}}$ Not available in $2 \mathrm{pPH}$ patients

${ }^{\mathrm{b}}$ Not available in $11 \mathrm{pPH}$ patients

end-diastolic volume, see Table 4 for values), RV GLS and all RA strain parameters remained significant (Table 5).

\section{Discussion}

In this study, we evaluated the alterations in RA and RV function using CMR-FT myocardial deformation in patients with $\mathrm{pPH}$. In general, patients with $\mathrm{pPH}$ had lower RVEF, longer RV contraction times, impaired RA strain, RV GLS, and RV GCS compared to healthy controls. More importantly, we identified changes in RA and RV strain even in $\mathrm{pPH}$ with preserved RVEF. RA reservoir, and especially conduit strain, was lower in this group of $\mathrm{pPH}$ patients. RV GLS was impaired, while RV GCS was preserved. This resulted in a higher CS/LS ratio, meaning that $\mathrm{RV}$ function was more dependent on circumferential shortening in pPH (Central Illustration). RV GLS and all RA phasic strain parameters were independently associated with MACE, beyond clinical (age, sex, WHO functional class $\geq 3$ ) and traditional imaging parameters (estimated RVSP, RVEF, and indexed-RV end-diastolic volume).

$\mathrm{RV}$ function is of utmost importance for a patient's prognosis in $\mathrm{pPH}[1,2]$. CMR is the gold standard for noninvasive measurement of global RV function, measuring volumes and calculating RVEF $[3,13]$. The recent development of CMR-FT makes it possible to measure RA and RV strain on standard cine images. In addition, inter- and intraobserver variability of CMR-FT is very low [14, 15], demonstrating good reproducibility. Echocardiography is widely available and inexpensive, and would therefore be the preferred method for measuring strain. However, a major drawback is the poor acoustic window, which limits RV strain imaging and lead to exclusion of data up to $12-17 \%[12,16]$. In our study, only one patient had to be excluded from CS measurement due to poor image quality. 
Table 3 CMR strain parameters of healthy controls \& precapillary pulmonary hypertension patients

\begin{tabular}{|c|c|c|c|}
\hline $\begin{array}{l}\text { Healthy controls } \\
(\mathrm{n}=20)\end{array}$ & $\mathrm{pPH}$ patients $(\mathrm{n}=45)$ & $\mathrm{P}$ value* & $\begin{array}{l}\text { pPH patients with preserved P value* } \\
\text { RVEF }(n=18)\end{array}$ \\
\hline
\end{tabular}

\begin{tabular}{|c|c|c|c|c|c|}
\hline \multicolumn{6}{|l|}{ Right atrial strain } \\
\hline Reservoir (\%) & $41 \pm 6$ & $29 \pm 10$ & $<0.001$ & $35 \pm 9$ & 0.02 \\
\hline Conduit (\%) & $23 \pm 5$ & $12 \pm 7$ & $<0.001$ & $16 \pm 8$ & 0.004 \\
\hline Booster (\%) & $18 \pm 4$ & $15 \pm 7$ & 0.10 & $19 \pm 8$ & 0.71 \\
\hline \multicolumn{6}{|l|}{ Right ventricular strain } \\
\hline Global LS (\%) & $-31 \pm 4$ & $-20 \pm 6$ & $<0.001$ & $-25 \pm 4$ & $<0.001$ \\
\hline TTP (as \% of whole cycle) & $40 \pm 4$ & $47 \pm 7$ & $<0.001$ & $48 \pm 8$ & $<0.001$ \\
\hline Free wall-LS (\%) & $-39 \pm 5$ & $-25 \pm 8$ & $<0.001$ & $-31 \pm 7$ & $<0.001$ \\
\hline Septal-LS (\%) & $-22 \pm 5$ & $-14 \pm 19$ & $<0.001$ & $-18 \pm 4$ & 0.01 \\
\hline $\begin{array}{l}\text { Free wall \& septum TTP differ- } \\
\text { ence }(\mathrm{ms})\end{array}$ & $0[0-0]$ & $26[-27-59]$ & 0.07 & $11[-27-29]$ & 0.55 \\
\hline Global CS $(\%)^{\mathrm{a}}$ & $-15 \pm 4$ & $-12 \pm 5$ & 0.01 & $-15 \pm 3$ & 0.80 \\
\hline Basal CS (\%) & $-12 \pm 3$ & $-12 \pm 4$ & 0.68 & $-15 \pm 3$ & 0.001 \\
\hline Mid CS (\%) & $-14 \pm 5$ & $-11 \pm 5$ & 0.04 & $-13 \pm 5$ & 0.67 \\
\hline Apical CS (\%) & $-21 \pm 7$ & $-13 \pm 9$ & 0.01 & $-17 \pm 7$ & 0.09 \\
\hline CS/LS ratio & $0.49 \pm 0.13$ & $0.58 \pm 0.24$ & 0.12 & $0.62 \pm 0.19$ & 0.02 \\
\hline
\end{tabular}

P-values in bold are statistically significant $(\mathrm{p}<0.05)$

Values are in means $\pm \mathrm{SD}$, medians [interquartile range], or $\mathrm{n}(\%)$

$C S$ circumferential strain, $L S$ longitudinal strain, $p P H$ precapillary pulmonary hypertension, $R A$ right atrium, $R V$ right ventricular, $T T P$ time to peak strain

*Compared to healthy controls

${ }^{a} \mathrm{pPH}$ patients $\mathrm{n}=44$ (exclusion of one patient due to insufficient quality of the short-axis cines)

The RV attempts to adapt to the increased pulmonary vascular resistance in $\mathrm{pPH}$ by increasing its contractility. $\mathrm{RV}$ remodeling, such as hypertrophy and changes in muscle properties, ensures that stroke volume can be maintained in the early stages. When these mechanisms fall short in later stages, the RV dilates and the heart rate increases, and RVEF decreases [1]. Since a decline in RVEF will only occur in later stages of heart failure, earlier markers of changed RV function would be valuable for guidance of medical therapy and follow-up of pPH patients. We believe that an impairment of RV FT-strain, recently shown to be of prognostic importance in other studies as well as ours [11, 17], might be such a marker. In addition, these parameters were able to identify changes in RV function, even in patients with preserved RVEF. In our study, RA conduit strain, reflecting passive filling of the RV, was lower in pPH patients with preserved RVEF compared to healthy controls. In a previous study by Tello et al. [18], RA strain was compared with invasive pressure-volume loop curves in $\mathrm{PH}$ patients, demonstrating that RA strain relates to RV end-diastolic pressure, reflecting diastolic function and stiffness of the RV, rather than RV contractility. This suggests that, similar to what is seen in the left ventricle [19], FT-strain is able to detect RV diastolic dysfunction.

RV GLS was also impaired in pPH patients with preserved RVEF, which is in line with previous studies, using fast strain-encoding imaging [20] as well as feature tracking derived strain $[11,17,21]$. CS is less frequently analyzed, but a recent study confirms our findings, demonstrating comparable GCS values in $\mathrm{PH}$ patients with preserved RVEF, and reduced GCS in PH patients with reduced RVEF [11]. This leads to higher CS/LS ratios in $\mathrm{pPH}$ patients in comparison to healthy controls. Under normal conditions, longitudinal shortening accounts for the majority of RV contraction [22]. It has been suggested that in $\mathrm{pPH}, \mathrm{RV}$ function might be more dependent on circumferential shortening. RV remodeling induces a relative dominance of hypertrophic circumferentially oriented fibers, and RV dilatation creates a more transverse fiber orientation [11, 23, 24]. In addition, segmental CS analysis demonstrated interesting results, showing preserved basal-CS, and reduced mid- and apical$\mathrm{CS}$ in pPH patients, the last being the most pronounced. This is similar to what is reported in a study of Kind et al. [23], measuring inward, radial movement, and a study of Fernandez-Friera et al. [25], measuring regional RVEF, both reporting apical function was most affected in $\mathrm{PH}$, and even present in PH patients with preserved RVEF [25]. This early apical dysfunction could be attributed to the apex being especially thin-walled. Hence, apical dilatation, and thus reduced function, is the first to show up when RV pressure increases, and is even seen in acute RV afterload elevation, such as acute pulmonary embolism [26]. 


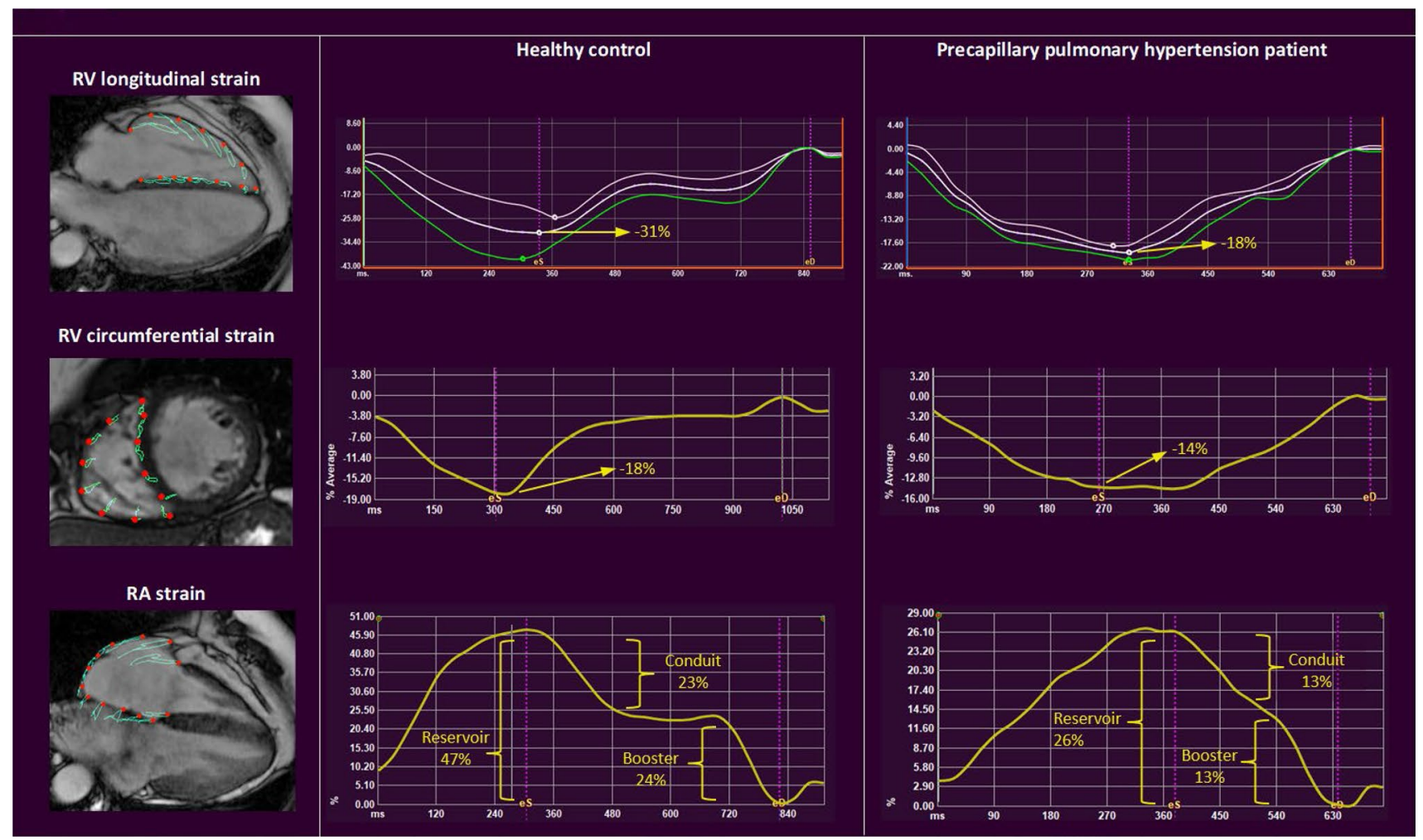

Fig. 2 Representative images of right ventricular longitudinal and circumferential strain, and right atrial strain in a healthy control and a precapillary pulmonary hypertension patient. Representative images of the different strain parameters (\%,y-axis) in time (ms, x-axis). Compared to the healthy controls, RV longitudinal (upper pictures)

\section{Clinical implications}

Although echocardiography is still the first line modality, CMR can provide incremental, relevant information in patients with proven $\mathrm{PH}$, as it is the gold standard for noninvasive measurement of RV function [3]. CMR-FT analysis can be performed on standard cine images in a few minutes with excellent reproducibility. In total, it would take about 10-15 min of CMR-acquisition time and 10 min for postprocessing analysis to obtain RA and RV volumes, calculate RVEF, and measure strain. These favorable characteristics make the implementation in clinical practice possible, e.g. for monitoring RA and RV function in follow-up of patients with PH. Future focus should focus on the clinical value of early RA and RV strain impairment-for guiding treatment strategy, prognosis, and possible improved screening for patients at risk of $\mathrm{pPH}$. and circumferential strain (middle pictures) are lower in pPH patients. In addition, RA reservoir and conduit strain are lower in $\mathrm{pPH}$ patients compared to healthy controls, whereas there were no significant differences in RA booster strain (lower pictures). $p P H$ pulmonary hypertension $R A$ right atrial, $R V$ right ventricular

\section{Limitations}

This study has limitations. This multicenter study, performed at tertiary hospitals, has a limited sample size, which could make findings less generalizable to the general population. Although inter- and intra-observer variability was excellent in our study, it is known that CMR-FT intersoftware variability exists [27]. RA and RV GLS were of prognostic value, independent of all evaluated clinical covariates. However, caution must be applied when interpreting these results, since due to the limited sample size, not all possible confounders could be assessed, such as the heterogeneity in treatment during follow-up. In addition, since the CMR was performed at a single timepoint, we can only speculate about the changes in RV function that arise over time, and larger prospective longitudinal studies are needed to further evaluate these patterns, and to evaluate their prognostic significance. 


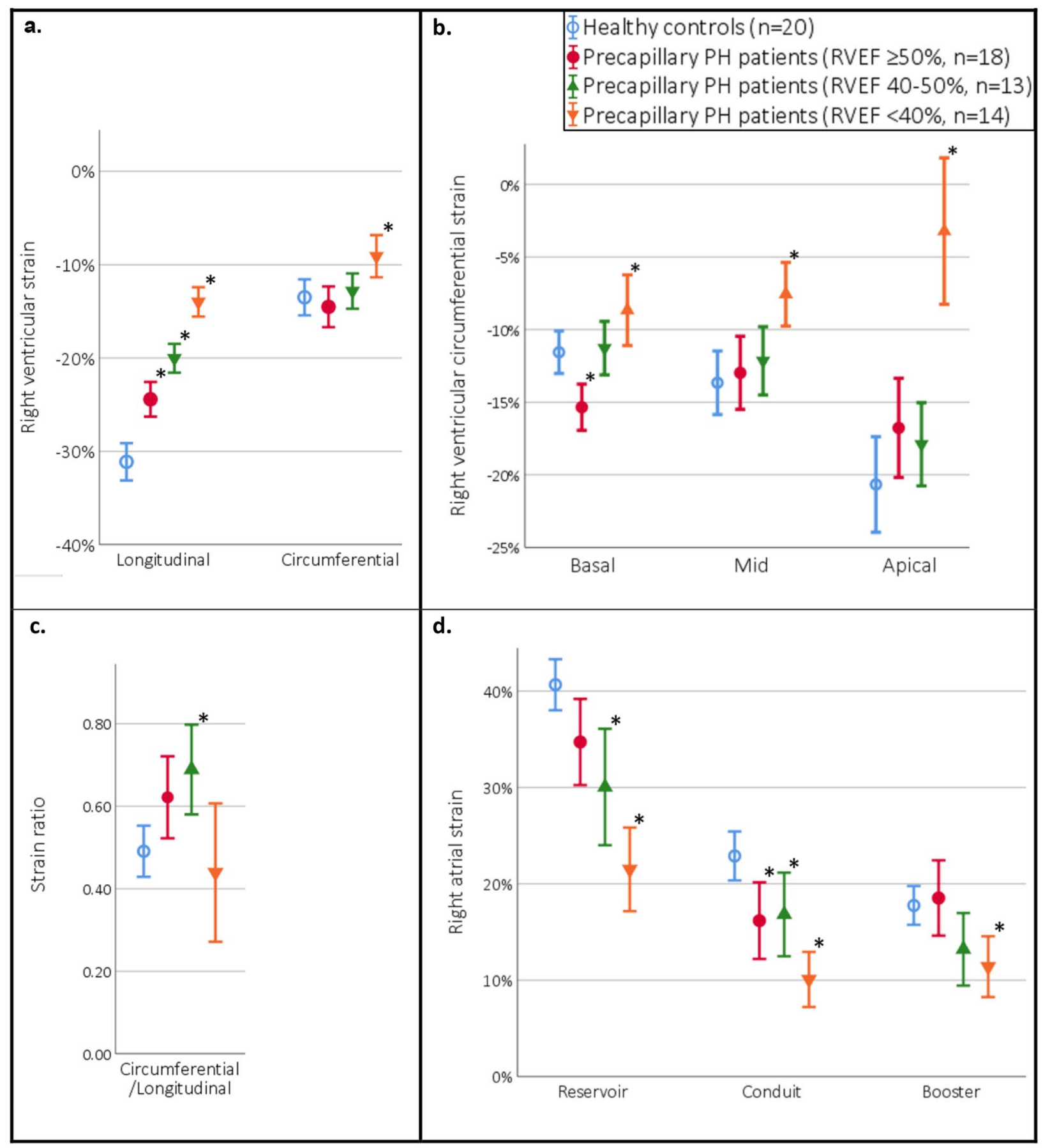

Fig. 3 Right ventricular longitudinal and circumferential strain, and right atrial strain in healthy controls and precapillary pulmonary hypertension patients (stratified in groups by right ventricular ejection fraction). a Right ventricular global longitudinal and global circumferential strain. Compared to healthy controls, all pPH patient groups had lower global longitudinal strain, whereas circumferential strain was only lower in the pPH patient group with $\mathrm{RVEF}<40 \%$. b Right ventricular basal, mid, and apical circumferential strain. In pPH patients with preserved RVEF $(\geq 50 \%)$ basal circumferential strain was better in comparison to healthy controls. In pPH patients with severely reduced RVEF $(<40 \%)$, circumferential strain was reduced on all levels. c Compared to healthy controls, patients with RVEF between 40 and $50 \%$ had a higher ratio between circumferential and longitudinal strain, in the other $\mathrm{pPH}$ patient groups no significant differences were found. d Right atrial strain. Compared to healthy controls, pPH patients had altered RA strain: all pPH patients had impaired conduit strain, and $\mathrm{pPH}$ patients with reduced RVEF $(<50 \%)$ had impaired reservoir strain. Booster strain was only significantly lower in $\mathrm{pPH}$ patient group with $\mathrm{RVEF}<40 \%$. $p P H$ precapillary pulmonary hypertension, $R V E F$ right ventricular ejection fraction. Bars are presented in means with error bars showing the $95 \%$ confidence intervals. $* \mathrm{p}<0.05$ compared to healthy controls 
Table 4 Univariable association with MACE

\begin{tabular}{llr}
\hline & pPH patients $(\mathrm{n}=33)$ & \\
\cline { 2 - 3 } & Hazard ratio $(95 \% \mathrm{CI})$ & P value \\
\hline Age (years) & $1.01(0.97-1.05)$ & 0.69 \\
Sex (male) & $1.02(0.31-3.42)$ & 0.96 \\
WHO functional class 3 or 4 & $3.66(1.15-11.71)$ & $\mathbf{0 . 0 3}$ \\
Estimated RVSP (mmHg) & $1.01(0.99-1.04)$ & 0.33 \\
RV end-diastolic volume-indexed $\left(\mathrm{mL} / \mathrm{m}^{2}\right)$ & $1.01(1.00-1.02)$ & $\mathbf{0 . 0 1}$ \\
RVEF $(\%)$ & $0.96(0.92-1.01)$ & 0.14 \\
RV global LS $(\%)^{\mathrm{a}}$ & $1.18(1.04-1.34)$ & $\mathbf{0 . 0 1}$ \\
RV global CS $(\%)^{\mathrm{a}}$ & $1.01(0.91-1.13)$ & 0.80 \\
RV CS Basal $(\%)^{\mathrm{a}}$ & $1.06(0.93-1.20)$ & 0.39 \\
RV CS Mid $(\%)^{\mathrm{a}}$ & $1.02(0.89-1.16)$ & 0.82 \\
RV CS Apical $(\%)^{\mathrm{a}}$ & $1.00(0.94-1.06)$ & 1.00 \\
Right atrial reservoir strain $(\%)$ & $0.88(0.83-0.95)$ & $<\mathbf{0 . 0 0 1}$ \\
Right atrial booster strain $(\%)$ & $0.86(0.76-0.96)$ & $\mathbf{0 . 0 0 7}$ \\
Right atrial conduit strain $(\%)$ & $0.85(0.75-0.97)$ & $\mathbf{0 . 0 2}$ \\
\hline
\end{tabular}

$\mathrm{P}$-values in bold are statistically significant $(\mathrm{p}<0.05)$

$C S$ circumferential strain, $L S$ longitudinal strain, $M A C E$ major adverse cardiovascular events, $R V$ right ventricular, $R V E F \mathrm{RV}$ ejection fraction, $R V S P \mathrm{RV}$ systolic pressure

${ }^{\mathrm{a}} \mathrm{Per}+1 \%$ strain increase (= less negative strain value, meaning strain becomes worse)

\section{Conclusions}

This study shows an impairment of RA and RV strain in pPH patients. More importantly, in pPH patients with a preserved RVEF, RA and RV strain parameters were able to identify changes in RV function, demonstrating altered RA strain and impaired RV GLS, whereas RV CS was preserved. This resulted in a higher CS/LS ratio, meaning that in $\mathrm{pPH}, \mathrm{RV}$ function was more dependent on circumferential shortening. In addition, RV LS and RA strain are independent predictors of MACE, beyond clinical and

Table 5 Adjusted model for the prediction of MACE (adjusted for NYHA class $\geq$ III and indexed RV end-diastolic volume)

\begin{tabular}{lll}
\hline & \multicolumn{2}{l}{ All patients $(\mathrm{n}=33)$} \\
\cline { 2 - 3 } & HR (95\% CI) & P value \\
\hline $\begin{array}{l}\text { RV global LS (per \% increase) } \\
\begin{array}{l}\text { Right atrial reservoir strain (per \% } \\
\text { increase) }\end{array}\end{array}$ & $1.18(1.04-1.34)$ & $\mathbf{0 . 0 1}$ \\
$\begin{array}{l}\text { Right atrial booster strain (per \% } \\
\text { increase) }\end{array}$ & $0.87(0.80-0.94)$ & $\mathbf{0 . 0 0 1}$ \\
$\begin{array}{l}\text { Right atrial conduit strain (per \% } \\
\text { increase) }\end{array}$ & $0.81(0.71-0.92)$ & $\mathbf{0 . 0 0 1}$ \\
\hline
\end{tabular}

$\mathrm{P}$-values in bold are statistically significant $(\mathrm{p}<0.05)$

$L S$ longitudinal strain, MACE major adverse cardiovascular events, $R V$ right ventricular

${ }^{\text {a }}$ Per $+1 \%$ strain increase (=less negative strain value, meaning strain becomes worse) imaging parameters. This study highlights the changes in contraction pattern that occur in pPH patients, even before global dysfunction is apparent. These results emphasize the incremental value of RA and RV strain analyses, to detect alterations in RV function even when RVEF is still preserved, and might be helpful to guide prognosis in $\mathrm{pPH}$ patients.

Supplementary Information The online version contains supplementary material available at https://doi.org/10.1007/s10554-022-02555-6.

Acknowledgements The authors thank Dr. A.L. Duijnhouwer and the technicians of the Cardiology and Radiology department of the University Medical Center Utrecht and Radboud University Medical Center for their skilled assistance.

Author contributions All authors contributed to the study conception and design. Material preparation, data collection and analysis were performed by JLV, and MMPD. The first draft of the manuscript was written by JLV and all authors commented on previous versions of the manuscript. All authors read and approved the final manuscript.

Funding Funding for this research was supported by a grant from ICIN-NHI, a non-commercial scientific institution (Utrecht, the Netherlands). This company was not involved in the writing, data analysis or conclusions of this manuscript. The funders had no role in study design, data collection and analysis, decision to publish, or preparation of the manuscript.

\section{Declarations}

Conflict of interest M.D. has received a research grant from the ICINNetherlands Heart House. R.N.: Received research grants from Biotronik and Philips. M.V.: Received research grants from Actelion (Fer- 
rer, United Therapeutics Therabel) and consulting fees from Actelion (Boehringer Ingelheim, Roche).

Ethical approval This study was performed in line with the principles of the Declaration of Helsinki. Approval was granted by the Medical Research Ethics Committee Utrecht and the Medical Ethics Committee of the Radboud University Medical Center (CMO region ArnhemNijmegen).

Informed consent Written informed consent was obtained from all individual participants included in the study.

Open Access This article is licensed under a Creative Commons Attribution 4.0 International License, which permits use, sharing, adaptation, distribution and reproduction in any medium or format, as long as you give appropriate credit to the original author(s) and the source, provide a link to the Creative Commons licence, and indicate if changes were made. The images or other third party material in this article are included in the article's Creative Commons licence, unless indicated otherwise in a credit line to the material. If material is not included in the article's Creative Commons licence and your intended use is not permitted by statutory regulation or exceeds the permitted use, you will need to obtain permission directly from the copyright holder. To view a copy of this licence, visit http://creativecommons.org/licenses/by/4.0/.

\section{References}

1. Vonk-Noordegraaf A, Haddad F, Chin KM, Forfia PR, Kawut SM, Lumens J, Naeije R, Newman J, Oudiz RJ, Provencher S, Torbicki A, Voelkel NF, Hassoun PM (2013) Right heart adaptation to pulmonary arterial hypertension: physiology and pathobiology. $\mathrm{J}$ Am Coll Cardiol 62(25 Suppl):D22-D33

2. Grapsa J, Pereira Nunes MC, Tan TC, Cabrita IZ, Coulter T, Smith BC, Dawson D, Gibbs JS, Nihoyannopoulos P (2015) Echocardiographic and hemodynamic predictors of survival in precapillary pulmonary hypertension: seven-year follow-up. Circ Cardiovasc Imaging 8(6): 002107

3. Galie N, Humbert M, Vachiery JL, Gibbs S, Lang I, Torbicki A, Simonneau G, Peacock A, Vonk Noordegraaf A, Beghetti M, Ghofrani A, Gomez Sanchez MA, Hansmann G, Klepetko W, Lancellotti P, Matucci M, McDonagh T, Pierard LA, Trindade PT, Zompatori M, Hoeper M (2016) 2015 ESC/ERS Guidelines for the diagnosis and treatment of pulmonary hypertension: The Joint Task Force for the Diagnosis and Treatment of Pulmonary Hypertension of the European Society of Cardiology (ESC) and the European Respiratory Society (ERS): endorsed by: Association for European Paediatric and Congenital Cardiology (AEPC), International Society for Heart and Lung Transplantation (ISHLT). Eur Heart J 37(1):67-119

4. Spruijt OA, Di Pasqua MC, Bogaard HJ, van der Bruggen CE, Oosterveer F, Marcus JT, Vonk-Noordegraaf A, Handoko ML (2017) Serial assessment of right ventricular systolic function in patients with precapillary pulmonary hypertension using simple echocardiographic parameters: a comparison with cardiac magnetic resonance imaging. J Cardiol 69(1):182-188

5. Pedrizzetti G, Claus P, Kilner PJ, Nagel E (2016) Principles of cardiovascular magnetic resonance feature tracking and echocardiographic speckle tracking for informed clinical use. J Cardiovasc Magn Reson Off J Soc Cardiovasc Magn Reson 18(1):51

6. Driessen MM, Baggen VJ, Freling HG, Pieper PG, van Dijk AP, Doevendans PA, Snijder RJ, Post MC, Meijboom FJ, Sieswerda GT, Leiner T, Willems TP (2014) Pressure overloaded right ventricles: a multicenter study on the importance of trabeculae in RV function measured by CMR. Int J Cardiovasc Imaging 30(3):599-608

7. Driessen MMP, Schings MA, Sieswerda GT, Doevendans PA, Hulzebos EH, Post MC, Snijder RJ, Westenberg JJM, van Dijk APJ, Meijboom FJ, Leiner T (2018) Tricuspid flow and regurgitation in congenital heart disease and pulmonary hypertension: comparison of 4D flow cardiovascular magnetic resonance and echocardiography. J Cardiovasc Magn Reson Off J Soc Cardiovasc Magn Reson 20(1):5

8. Rudski LG, Lai WW, Afilalo J, Hua L, Handschumacher MD, Chandrasekaran K, Solomon SD, Louie EK, Schiller NB (2010) Guidelines for the echocardiographic assessment of the right heart in adults: a report from the American Society of Echocardiography endorsed by the European Association of Echocardiography, a registered branch of the European Society of Cardiology, and the Canadian Society of Echocardiography. J Am Soc Echocardiogr Off Publ Am Soc Echocardiogr 23(7):685-713

9. Leng S, Tan RS, Zhao X, Allen JC, Koh AS, Zhong L (2018) Validation of a rapid semi-automated method to assess left atrial longitudinal phasic strains on cine cardiovascular magnetic resonance imaging. J Cardiovasc Magn Reson Off J Soc Cardiovasc Magn Reson 20(1):71

10. Maceira AM, Prasad SK, Khan M, Pennell DJ (2006) Reference right ventricular systolic and diastolic function normalized to age, gender and body surface area from steady-state free precession cardiovascular magnetic resonance. Eur Heart J 27(23):2879-2888

11. de Siqueira ME, Pozo E, Fernandes VR, Sengupta PP, Modesto K, Gupta SS, Barbeito-Caamano C, Narula J, Fuster V, Caixeta A, Sanz J (2016) Characterization and clinical significance of right ventricular mechanics in pulmonary hypertension evaluated with cardiovascular magnetic resonance feature tracking. J Cardiovasc Magn Reson Off J Soc Cardiovasc Magn Reson 18(1):39

12. Sachdev A, Villarraga HR, Frantz RP, McGoon MD, Hsiao JF, Maalouf JF, Ammash NM, McCully RB, Miller FA, Pellikka PA, Oh JK, Kane GC (2011) Right ventricular strain for prediction of survival in patients with pulmonary arterial hypertension. Chest 139(6):1299-1309

13. Kiely DG, Levin D, Hassoun P, Ivy DD, Jone PN, Bwika J, Kawut SM, Lordan J, Lungu A, Mazurek J, Moledina S, Olschewski H, Peacock A, Puri GD, Rahaghi F, Schafer M, Schiebler M, Screaton N, Tawhai M, Van Beek EJ, Vonk-Noordegraaf A, Vanderpool RR, Wort J, Zhao L, Wild J, Vogel-Claussen J, Swift AJ (2019) EXPRESS: statement on imaging and pulmonary hypertension from the Pulmonary Vascular Research Institute (PVRI). Pulm Circ 9(3):2045894019841990

14. Truong VT, Safdar KS, Kalra DK, Gao X, Ambach S, Taylor MD, Moore R, Taylor RJ, Germann J, Toro-Salazar O, Jefferies JL, Bartone C, Raman SV, Ngo T, Mazur W (2017) Cardiac magnetic resonance tissue tracking in right ventricle: feasibility and normal values. Magn Reson Imaging 38:189-195

15. Peng J, Zhao X, Zhao L, Fan Z, Wang Z, Chen H, Leng S, Allen J, Tan RS, Koh AS, Ma X, Lou M, Zhong L (2018) Normal values of myocardial deformation assessed by cardiovascular magnetic resonance feature tracking in a healthy chinese population: a multicenter study. Front Physiol 9:1181

16. Fine NM, Chen L, Bastiansen PM, Frantz RP, Pellikka PA, Oh JK, Kane GC (2013) Outcome prediction by quantitative right ventricular function assessment in 575 subjects evaluated for pulmonary hypertension. Circ Cardiovasc Imaging 6(5):711-721

17. Kallifatidis A, Mouratoglou SA, Giannakoulas G, Finitsis S, Karvounis H, Sianos G (2020) Myocardial deformation assessment in patients with precapillary pulmonary hypertension: a cardiac magnetic resonance study. Diagn Interv Imaging 102:153-161 
18. Tello K, Dalmer A, Vanderpool R, Ghofrani HA, Naeije R, Roller F, Seeger W, Wiegand M, Gall H, Richter MJ (2020) Right ventricular function correlates of right atrial strain in pulmonary hypertension: a combined cardiac magnetic resonance and conductance catheter study. Am J Physiol Heart Circ Physiol 318(1):H156-H164

19. Kammerlander AA, Kraiger JA, Nitsche C, Donà C, Duca F, Zotter-Tufaro C, Binder C, Aschauer S, Loewe C, Hengstenberg C, Bonderman D, Mascherbauer J (2019) Global longitudinal strain by CMR feature tracking is associated with outcome in HFPEF. JACC Cardiovasc Imaging 12(8 Pt 1):1585-1587

20. Shehata ML, Harouni AA, Skrok J, Basha TA, Boyce D, Lechtzin N, Mathai SC, Girgis R, Osman NF, Lima JA, Bluemke DA, Hassoun PM, Vogel-Claussen J (2013) Regional and global biventricular function in pulmonary arterial hypertension: a cardiac MR imaging study. Radiology 266(1):114-122

21. Lindholm A, Hesselstrand R, Radegran G, Arheden H, Ostenfeld E (2018) Decreased biventricular longitudinal strain in patients with systemic sclerosis is mainly caused by pulmonary hypertension and not by systemic sclerosis per se. Clin Physiol Funct Imaging 39:215-225

22. Sanz J, Sanchez-Quintana D, Bossone E, Bogaard HJ, Naeije R (2019) Anatomy, function, and dysfunction of the right ventricle: JACC state-of-the-art review. J Am Coll Cardiol 73(12):1463-1482

23. Kind T, Mauritz GJ, Marcus JT, van de Veerdonk M, Westerhof N, Vonk-Noordegraaf A (2010) Right ventricular ejection fraction is better reflected by transverse rather than longitudinal wall motion in pulmonary hypertension. J Cardiovasc Magn Reson Off J Soc Cardiovasc Magn Reson 12:35

24. Kovács A, Lakatos B, Tokodi M, Merkely B (2019) Right ventricular mechanical pattern in health and disease: beyond longitudinal shortening. Heart Fail Rev 24(4):511-520

25. Fernandez-Friera L, Garcia-Alvarez A, Guzman G, Bagheriannejad-Esfahani F, Malick W, Nair A, Fuster V, Garcia MJ, Sanz J (2011) Apical right ventricular dysfunction in patients with pulmonary hypertension demonstrated with magnetic resonance. Heart (Br Cardiac Soc) 97(15):1250-1256

26. McConnell MV, Solomon SD, Rayan ME, Come PC, Goldhaber SZ, Lee RT (1996) Regional right ventricular dysfunction detected by echocardiography in acute pulmonary embolism. Am J Cardiol 78(4):469-473

27. Bourfiss M, Vigneault DM, Aliyari Ghasebeh M, Murray B, James CA, Tichnell C, Mohamed Hoesein FA, Zimmerman SL, Kamel IR, Calkins H, Tandri H, Velthuis BK, Bluemke DA, Te Riele A (2017) Feature tracking CMR reveals abnormal strain in preclinical arrhythmogenic right ventricular dysplasia/cardiomyopathy: a multisoftware feasibility and clinical implementation study. J Cardiovasc Magn Reson Off J Soc Cardiovasc Magn Reson 19(1):66

Publisher's Note Springer Nature remains neutral with regard to jurisdictional claims in published maps and institutional affiliations. 